\title{
Measurement Based Power System Dynamics Prediction with Multivariate AutoRegressive Model
}

\author{
Changgang Li, Yong Liu, Kai Sun, Yilu Liu \\ Dept. of Electrical Engineering and Computer Science \\ University of Tennessee Knoxville \\ Knoxville, TN, US \\ cli27@utk.edu,yliu66@utk.edu, kaisun@utk.edu, \\ liu@utk.edu
}

\author{
Navin Bhatt \\ Electric Power Research Institute (EPRI) \\ Knoxville, TN, US \\ nbhatt@epri.com
}

\begin{abstract}
Power system dynamics prediction is important for online situation awareness and adaptive control. Model-based simulation is not applicable for dynamics prediction due to constant change of network configuration. With more and more Phasor Measurement Units (PMU), power system dynamics can be studied with pure measurement data. This paper proposes a dynamics prediction method based on measurement data. A mulit-input multi-output Multivariate AutoRegressive model (MAR) is developed and dynamics prediction procedure is proposed. Both simulation data and field measurement data are tested. Examples show that the proposed method can predict power system dynamics with high accuracy for simulation data and give reasonable result for measurement data. It provides an alternative approach to study power system dynamics.

Index Terms-Power systems, dynamics prediction, phasor measurement, multivariate autoregressive
\end{abstract}

\section{INTRODUCTION}

$\mathrm{P}$ OWER system is subjected to disturbances, small or large, at all times. In most cases, disturbances are limited in a small area and cleared quickly with modern control strategies. Power system can return to original operation point or transit to a new stable operation point in a short time. Some severe disturbances, however, may cause significant voltage or frequency deviation, and even lead to system instability. To prevent system collapse, it is desirable to provide system operators with early situation awareness of those severe cases. Besides, most response-driven control strategies, e.g., UnderFrequency Load Shedding (UFLS), act only when preset criterion is violated. Its adaptability can be improved by predicting dynamic response in advance and triggering control strategies before criterion is violated. A good dynamics prediction method is required to fulfill these objectives.

Time-domain simulation based on models of system equipment is a sophisticated method for dynamics analysis [1], and there are some commercial packages available, e.g.,

This work was supported by Electric Power Research Institute and also made use of Engineering Research Center Shared Facilities supported by the Engineering Research Center Program of the National Science Foundation and DOE under NSF Award Number EEC1041877 and the CURENT Industry Partnership Program.
PSS/E, DigSILENT, etc. A good time-domain simulation should be performed with good model of study system and well-defined disturbance description. However, power system model is not accurate enough especially for load side. With constantly changing power system topology and operation status, it is not feasible to update power system models in real time and the result of model-based simulation is not fully reliable. Meanwhile, it is hard to estimate disturbance information online. Without accurate model and disturbance information, model-based simulation can hardly be used for online dynamics prediction.

Benefited from accurate timing technology of Global Positioning System (GPS), Phasor Measurement Units (PMU) is developed to monitor system dynamics with high accuracy [2]. Since PMU data are calculated from the directly measured voltage and current waveforms, PMU is more reliable than model-based simulation in the sense of authenticity. Without knowledge of system parameters, some papers propose dynamics response prediction methods based on polynomial model [3]-[4], trigonometric functions [5], and reduced system model [6]-[7]. AutoRegressive (AR) model is used in [8] for instability prediction. However, it uses only dynamics of one measurement signal and is not systematical. Artificial intelligent based methods are also reported for dynamics prediction and stability prediction [9]-[10].

This paper proposes a dynamics prediction method with linear multivariate autoregressive (MAR) model based on pure measurement data. It is organized as follows. In section II, some basic concepts of system identification is introduced and the MAR model is discussed. Dynamics prediction procedure is proposed in section III. Some prediction examples are given with both simulation data and field measurement data in section IV. Conclusions are drawn in section V.

\section{System IDENTIFICATION AND Multivariate AUTOREGRESSIVE MODEL}

\section{A. General Model of Linear Time-Invariant Systems}

There are mainly two ways to build mathematical model for 
a dynamic system which can be represented with a series of differential and/or algebraic equations. One way is well known as "modeling" with which power system can be modeled by combinations of different simple blocks if the mechanism of all sub-systems and equipment is well studied. Model-based power system analysis software, e.g., PSS/E, are developed with models built in this way. The other way is "system identification" (SI) which is based on measurement signals. In this way, power system can be treated as a black box with finite observation points, i.e., PMUs, Frequency Disturbance Recorders (FDR)[11]. The measurement-based model built with SI is usually expressed in a transfer function form.

For a linear time-invariant system sampled at a time interval of $h$, a general SI transfer function structure is [12]

$$
A(q) y(t)=\frac{B(q)}{F(q)} u(t)+\frac{C(q)}{D(q)} e(t)
$$

where

$$
\begin{aligned}
& A(q)=1+a_{1} q^{-1}+\ldots+a_{n_{a}} q^{-n_{a}}, B(q)=b_{1} q^{-1}+\ldots+b_{n_{b}} q^{-n_{b}} \\
& C(q)=1+c_{1} q^{-1}+\ldots+c_{n_{c}} q^{-n_{c}}, D(q)=1+d_{1} q^{-1}+\ldots+d_{n_{d}} q^{-n_{d}} \\
& F(q)=1+f_{1} q^{-1}+\ldots+f_{n_{f}} q^{-n_{f}}
\end{aligned}
$$

Here, $y(t)$ is output signal which is the observable (measurable) system response of interest, $u(t)$ is input signal which is a stimuli manipulated by observer and affecting output signals, and $e(t)$ is a sequence of independent random variables. $n_{a}, n_{b}, n_{c}, n_{d}$, and $n_{f}$ are order of each part. $q^{-1}$ is a backward shift operator and $q^{-1} y(t)=y(t-h)$.

For a physical system, except for output signals and input signals, there is another kind of signal called "disturbance signals" and can be divided into two parts: those are directly measured and those are unmeasured but observable through output signals. Measured disturbance signal is mathematically the same as input signal and can be treated as a special input signal. Other disturbance signals are usually modeled with random sequence $e(t)$. It should be noted here that, the "disturbance signal" used for SI is different from power system "disturbances". To avoid confusion about "disturbance signal" and power system "disturbances", we use "events" instead of "disturbances" in the following parts to describe incidents changing power system, e.g., load increase, generation trip.

With different combinations of signals, different transfer function models can be developed. For example, if $B(q)=1$, $F(q)=1, C(q)=1$ and $D(q)=1,(1)$ is a univariate AR model used in [8]. If $C(q)=1, D(q)=1$ and $F(q)=1, \quad(1)$ becomes AutoRegressive model with eXogenous inputs (ARX).

\section{B. Single Output Multivariate AutoRegressive Model}

For power systems, we usually focus on dynamics response of frequency, voltage and angle under events. Those measured signals from PMUs or FDRs can be chosen as output signals. However, due to the diversity of events, the disturbance signal (or input signal) is hard to measure. In this sense, it is difficult to develop a transfer function model as defined in (1) with input signals. As in [8], AR model is usually used with only measurement output signals. To develop a systematic model describing the relationship between measurement signals, an alternative way is used with the basic idea of treating some measured signals as input signals.

With $N$ measured signals $y_{1}(t), \ldots, y_{N}(t)$, if we treat $y_{i}(t)$ as output signal and all other signals as input signals, a transfer function with $C(q)=1, D(q)=1$ and $F(q)=1$ can be developed

$$
A_{i}(q) y_{i}(t)=\sum_{j=1,1, j \neq i}^{N} B_{i j}(q) y_{j}(t)+e_{i}(t)
$$

It is similar to ARX model except the input signals are measured response which can be also treated as output signals. It is called multi-input single-output (MISO) Multivariate AutoRegressive model (MAR). Expand (2) yields

$$
\begin{aligned}
y_{i}(t)+a_{i 1} y_{i}(t-h)+\ldots+a_{i n_{a i}} y_{i}\left(t-n_{a i} h\right)= \\
\quad \sum_{j=1, j \neq i}^{N}\left[b_{i j 1} y_{j}(t-h)+\ldots+b_{i j n_{b j i}} y_{j}\left(t-n_{b j i} h\right)\right]+e_{i}(t)
\end{aligned}
$$

where $n_{a i}$ is the order of signal $y_{i}(t)$ and $n_{b j i}$ is the order of signal $y_{j}(t)$ when $y_{i}(t)$ is output signal and $y_{j}(t)$ is input signal.

In the MAR model, the orders of each signal can be different. For simplicity, we choose a uniform order $n$ for all signals whenever they are treated as input signal or output signal. With this manipulation, (3) can be rewritten as

$$
\begin{aligned}
& y_{i}(t)+a_{i 1} y_{i}(t-h)+\ldots+a_{i n} y_{i}(t-n h)= \\
& \quad \sum_{j=1, j \neq i}^{N}\left[b_{i j 1} y_{j}(t-h)+\ldots+b_{i j n} y_{j}(t-n h)\right]+e_{i}(t)
\end{aligned}
$$

or

$$
\begin{aligned}
y_{i}(t) & =b_{i i 1} y_{i}(t-h)+\ldots+b_{i i n} y_{i}(t-n h) \\
& \sum_{j=1, j \neq i}^{N}\left[b_{i j 1} y_{j}(t-h)+\ldots+b_{i j n} y_{j}(t-n h)\right]+e_{i}(t)
\end{aligned}
$$

where $b_{i i k}=-a_{i k}, k=1, . ., n$.

Equation (5) can be further expressed in vector form as

$$
y_{i}(t)=\sum_{j=1}^{N} \mathbf{b}_{i j} \mathbf{y}_{j}(t)+e_{i}(t)
$$

or

$$
y_{i}(t)=\sum_{j=1}^{N} \mathbf{y}_{j}(t)^{T} \mathbf{b}_{i j}{ }^{T}+e_{i}(t)
$$

where

$$
\mathbf{b}_{i j}=\left[b_{i j 1}, \ldots, b_{i j n}\right], \mathbf{y}_{j}(t)=\left[y_{j}(t-h), \ldots, y_{j}(t-n h)\right]^{T}
$$

and superscript $T$ indicates transpose.

For event with $m$ measurement data points, $m-n$ equations can be written as (8).

$$
\begin{aligned}
y_{i}((n+1) h) & =\sum_{j=1}^{N} \mathbf{y}_{j}((n+1) h)^{T} \mathbf{b}_{i j}{ }^{T}+e_{i}((n+1) h) \\
& \cdots \\
y_{i}(m h) & =\sum_{j=1}^{N} \mathbf{y}_{j}(m h)^{T} \mathbf{b}_{i j}{ }^{T}+e_{i}(m h)
\end{aligned}
$$

or

$$
\left[\begin{array}{c}
y_{i}((n+1) h) \\
\ldots \\
y_{i}(m h)
\end{array}\right]=\left[\begin{array}{ccc}
\mathbf{y}_{1}((n+1) h)^{T} & \ldots & \mathbf{y}_{N}((n+1) h)^{T} \\
\ldots & \ldots & \ldots \\
\mathbf{y}_{1}(m h)^{T} & \ldots & \mathbf{y}_{N}(m h)^{T}
\end{array}\right]\left[\begin{array}{l}
\mathbf{b}_{i 1}{ }^{T} \\
\ldots \\
\mathbf{b}_{i N}{ }^{T}
\end{array}\right]+\left[\begin{array}{l}
e_{i}((n+1) h) \\
\ldots \\
e_{i}(m h)
\end{array}\right]
$$

Since all those equations are linear, it can be solved with 
least square error estimator to minimize the $e_{i}(t)$ part. The model accuracy is greatly affected by model order $n$. Generally, optimum $n$ increases with the increase of PMU reporting rate. In practice, $n$ lies in the range from 10 to 30 . Low order can reduce prediction accuracy while too high model order can cause overtraining. The optimum model order should be optimized as an additional parameter of (9) according to system configuration.

\section{Signal Detrending}

Trend in a time series is a slow, gradual change in some property of the series over the whole study time window. For SI, all signals should be detrended before used to train the transfer function. There are different detrending methods such as first differencing, curve fitting, digital fitting. In most cases, first differencing is good enough, and is used in this paper. It is defined as

$$
y_{i}(t)=x_{i}(t)-x_{i}(t-h)
$$

where $x_{i}(t)$ is measurement signal, and $y_{i}(t)$ is detrended signal.

For dynamics study, we focus on the dynamics of original signal $x_{i}(t)$ instead of detrended signal $y_{i}(t)$. To recover the original signal from detrended signal, we can get the inverse form of first differencing filter as

$$
x_{i}(t)=y_{i}(t)+x_{i}(t-h)
$$

It is clear that the recovery equation (11) depends on the current detrended signal $y_{i}(t)$ and historical data $x_{i}(t-h)$ which is already know at time $t$. So the detrending method used can be easily implemented for field application.

\section{DyNAMics PREDICTION wITH MAR MODEL}

\section{A. Multi Output Multivariate AutoRegressive Model}

In the MAR model, there is no difference between the output signal $y_{i}(t)$ and other signals in the respect of mathematical status. We can develop MAR models with other signals as output signal as follows

$$
\begin{gathered}
y_{1}(t)=\sum_{j=1}^{N} \mathbf{b}_{1 j} \mathbf{y}_{j}(t)+e_{1}(t) \\
\cdots \\
y_{N}(t)=\sum_{j=1}^{N} \mathbf{b}_{N j} \mathbf{y}_{j}(t)+e_{N}(t)
\end{gathered}
$$

Let $\mathbf{y}(t)=\left[y_{1}(t), \ldots, y_{N}(t)\right]^{T}$, we can get the multi-input multi-output (MIMO) MAR model as follows

$$
\mathbf{y}(t)=\left[\begin{array}{ccc}
\mathbf{b}_{11} & \ldots & \mathbf{b}_{1 N} \\
\ldots & \ldots & \ldots \\
\mathbf{b}_{N 1} & \ldots & \mathbf{b}_{N N}
\end{array}\right]\left[\begin{array}{c}
\mathbf{y}_{1}(t) \\
\ldots \\
\mathbf{y}_{N}(t)
\end{array}\right]+\left[\begin{array}{c}
e_{1}(t) \\
\ldots \\
e_{N}(t)
\end{array}\right]
$$

Unlike the univariate AR model used in [8], the MIMO MAR model describes the interaction between different measurements. Theoretically, all quantities of power system are affected by other quantities. More measurement signals would improve prediction accuracy and, however, increase model complexity. To reduce model complexity, some input signals with low correlation with output signal can be omitted.
How to reduce input/output complexity is beyond the scope of this paper and all available measurement signals are used in examples of next section as a general approach.

\section{B. Prediction Procedure}

In equation (1), the random part $e(t)$ corresponds to the unmeasured disturbance signals. However, for power system dynamics, the unmeasured dynamics is not a random process. It is hard to model $e_{i}(t)$ in (12). For simplicity, the $e_{i}(t)$ part is neglected and the MAR model becomes

$$
\mathbf{y}(t)=\left[\begin{array}{ccc}
\mathbf{b}_{11} & \ldots & \mathbf{b}_{1 N} \\
\ldots & \ldots & \ldots \\
\mathbf{b}_{N 1} & \ldots & \mathbf{b}_{N N}
\end{array}\right]\left[\begin{array}{c}
\mathbf{y}_{1}(t) \\
\ldots \\
\mathbf{y}_{N}(t)
\end{array}\right]
$$

or

$$
\mathbf{y}(t)=\mathbf{B Y}(t)
$$

where

$$
\mathbf{B}=\left[\begin{array}{ccc}
\mathbf{b}_{11} & \ldots & \mathbf{b}_{1 N} \\
\ldots & \ldots & \ldots \\
\mathbf{b}_{N 1} & \ldots & \mathbf{b}_{N N}
\end{array}\right]
$$

and

$$
\mathbf{Y}(t)=\left[\begin{array}{lll}
\mathbf{y}_{1}(t)^{T} & \ldots & \mathbf{y}_{N}(t)^{T}
\end{array}\right]^{T}
$$

Once the MAR model is trained, dynamics can be predicted recursively with the flowchart in Fig. 1 where $t_{\max }$ is the length of study time window. Since $e_{i}(t)$ part is neglected, the prediction error may accumulate as $t$ increases. The accuracy of predicted response is examined in next section.

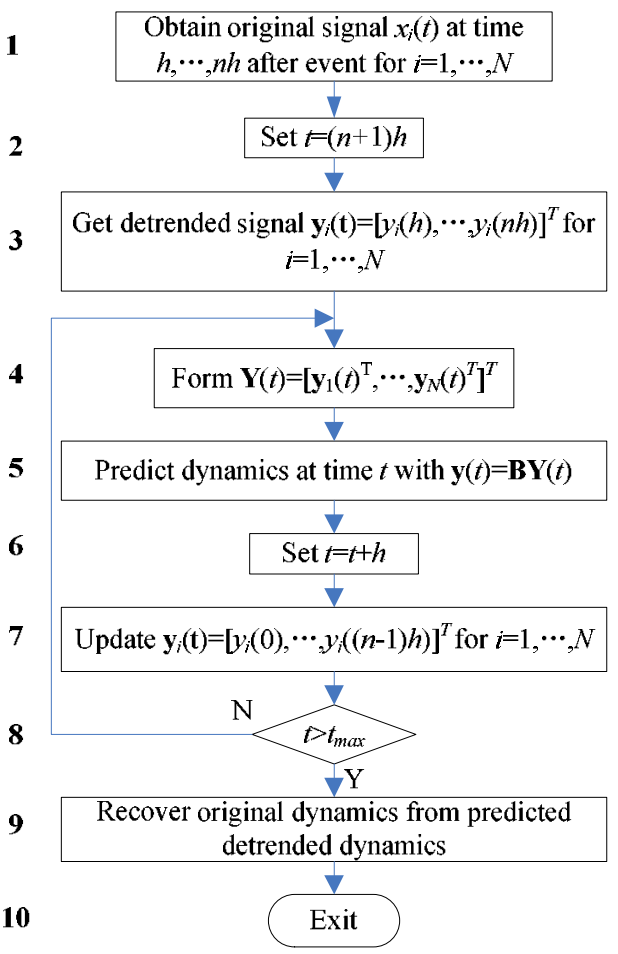

Fig.1 Flowchart of dynamics prediction with MAR model

It can be seen from Fig. 1 that the MAR model requires $n$ data point for prediction. The first $n$ points after event (as in 
step 1) contain important information of the event, and trigger the following dynamics. There should be only one event or operation during the $n$ points. Events with more than one operation, e.g., line fault which requires fault operation and clearing operation, cannot be modeled with MAR model.

It should be noted that the MAR model used in this paper is a linear model. It can be used for dynamics study of events that are relatively small comparing with system capacity, e.g., load increase or decrease, generation trip. Line fault that will cause topology change is out of the scope of the linear model. So, the linear MAR model targets small signal stability.

\section{DYNAMICS PREDICTION EXAMPLES}

To verify the proposed dynamics prediction method, model-based simulation data and field measurement data are used to demonstrate its performance in this section.

\section{A. Model-Based Simulation Data}

A 23-bus system is chosen as test system. Its data can be found in PSS/E manual. Simulation time step is set as half cycle (1/120s), but the output data is down sampled to mimic PMUs with $60 \mathrm{~Hz}$ output rate. Frequency, voltage and angle of all 17 PQ buses are monitored. In the following results, MAR model is always trained with data under $1 \%$ load increase event. All predictions are made for 5\% load increase event. The order of each signal is 13 .

(1) Voltage dynamics

For voltage prediction, a MAR model is trained with voltage dynamics of 17 buses. The predicted voltage dynamics of bus 151 is shown in Fig. 2. Blue diamond indicates where the prediction starts from. Data before the diamond are historical data and data after the diamond are predicted.

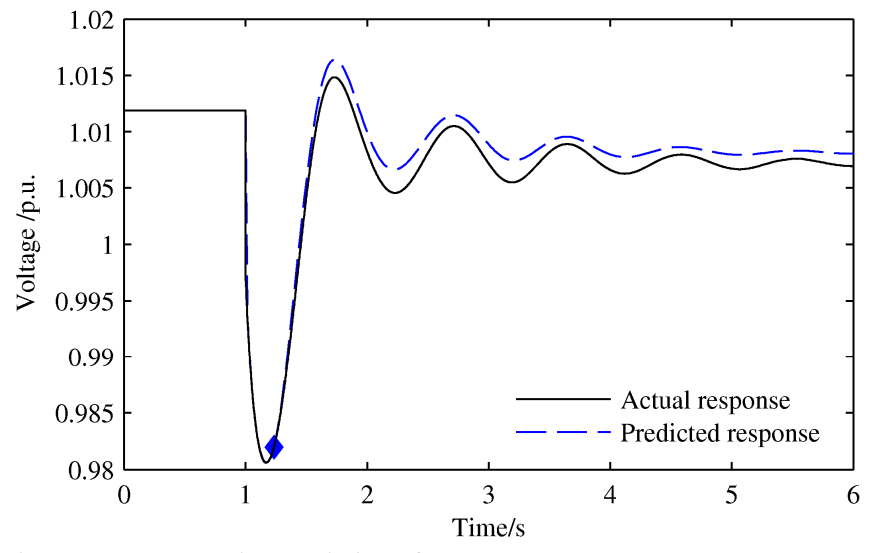

Fig.2 Voltage dynamics prediction of 23-bus system

(2) Angle difference between bus 151 and 3008

For angle prediction, a MAR model is trained with angle dynamics of 17 buses. Fig. 3 shows the predicted angle difference between bus 151 and 3008 .

(3)Frequency dynamics

For frequency prediction, a MAR model is trained with frequency dynamics of 17 buses. Fig. 4 shows the predicted frequency dynamics of bus 151 .

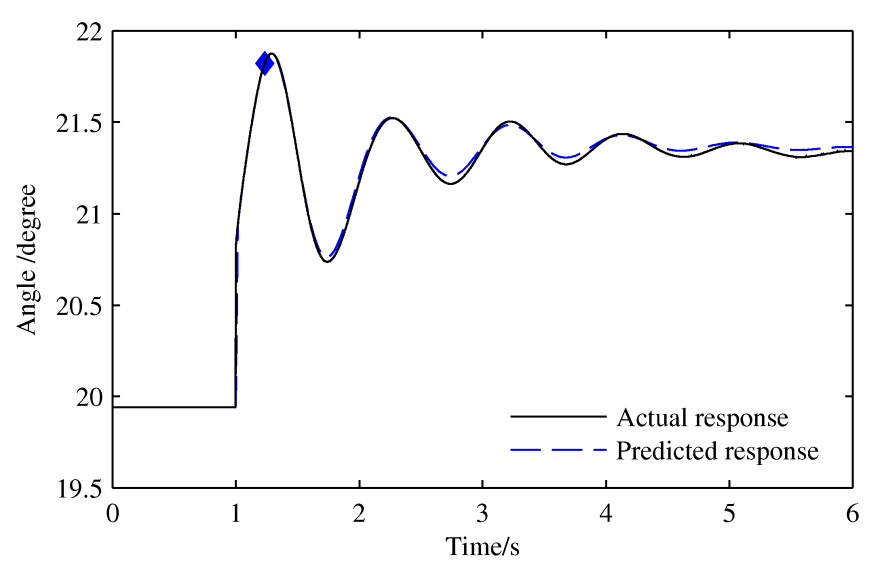

Fig.3 Angle difference prediction of 23-bus system

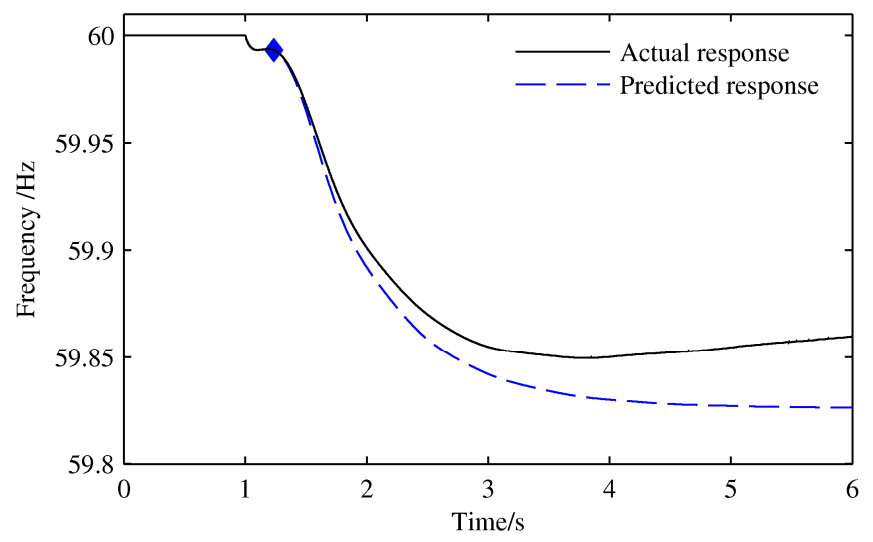

Fig.4 Frequency dynamics prediction of 23-bus system

From Fig. 2 and Fig. 3, it can be seen that the predicted voltage and angle response is very accurate comparing with actual response. But the frequency prediction in Fig. 4 is not as good as voltage and angle since the frequency dynamics is monotonous.

It can be also found from the three figures that predicted dynamics is more accurate in the first few tens of cycles than latter parts which is mainly caused by the neglect of $e_{i}(t)$ part.

\section{B. Field Measurement Data}

With a generation trip at 22:52:00 on July 1, 2012 near ISO New England of US, frequency, voltage and angle dynamics of 7 PMUs are measured. A MAR models is built with frequency and angle dynamics for frequency and angle prediction. Another MAR model is built with angle and voltage dynamics for voltage prediction. The order of each signal is 14 . The MAR models are tested with another generation trip event at 22:41:00 on Oct. 29, 2012 near ISO New England. The predicted frequency, angle difference and voltage dynamics are shown in Fig. 5 to Fig. 7.

From Fig. 5 to Fig. 7, it can be found that the prediction result with field measurement data is not as good as modelbased simulations. There are mainly 3 reasons. The first reason is that we have only 7 PMUs which are not enough for model training. The second reason is that real power system is subjected to perturbations all the time such as load change which makes the real response more complex than single event. 
The third reason is that raw PMU data is not as pure as simulation data. Though the noisy PMU data is filtered with a median filter in this paper, it is still not perfect for dynamics prediction. The noisy PMU data and neglect of $e_{i}(t)$ deteriorate the performance of the prediction method.

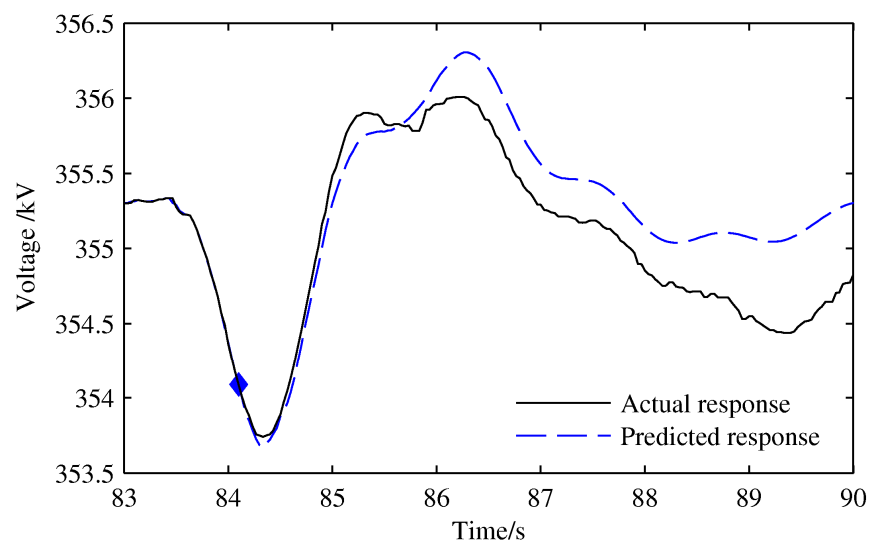

Fig.5 Voltage dynamics prediction of ISO-NE

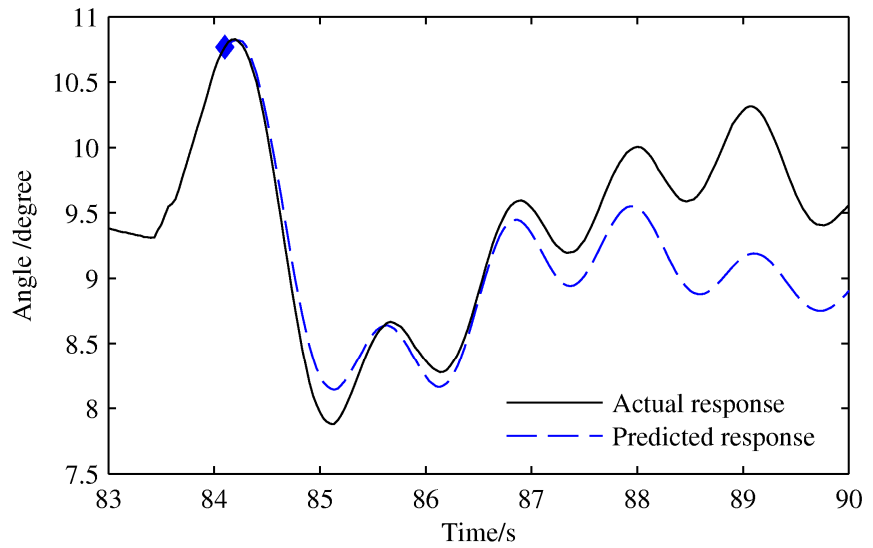

Fig.6 Angle difference dynamics prediction of ISO-NE

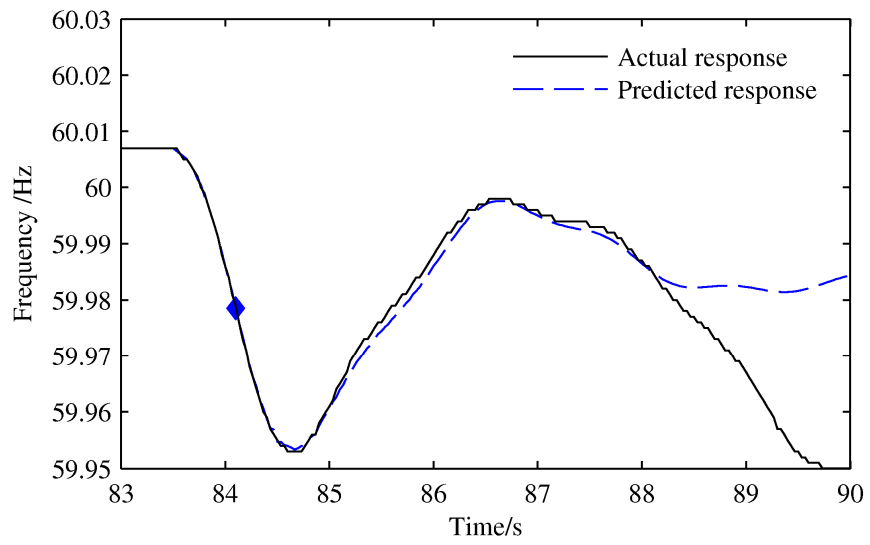

Fig.7 Frequency dynamics prediction of ISO-NE

Though the result of Fig. 5 to Fig. 7 is not very accurate, the trend of voltage, angle and frequency dynamics can still be predicted. The first 3-4 seconds of voltage and frequency prediction is reliable and predicted angle dynamics preserves predominant oscillation modes. More works need to be done with field measurement data including data filtering technology.

\section{CONCLUSIONS}

With more and more PMUs, massive measurement data gives an alternative approach to study power system dynamics with real response. The dynamics prediction method proposed in this paper is purely measurement-based and the MAR model can be easily updated online with typical events. Examples show that the accuracy of prediction is high for simulation cases. Real measurement case is not as accurate as simulation case, but still provides good prediction result for the first few seconds. Future works are to study how to choose the best measurement set, decide the best combinations of input/output signals while reducing input/output complexity and improve its accuracy for situation awareness and adaptive control.

\section{ACKNOWLEDGEMENT}

The authors gratefully acknowledge ISO New England for providing PMU measurement data and FNET team of University of Tennessee Knoxville for in-depth discussion on this topic.

\section{REFERENCES}

[1] Prabha Kundur, "Power System Stability and Control", New York: McGraw-Hill, Inc. 1994

[2] J. De La Ree, V. Centeno, J. S. Thorp, A. G. Phadke. "Synchronized Phasor Measurement Applications in Power Systems". IEEE Trans. on Smart Grid, vol. 1, no. 1, pp. 20-27, 2010.

[3] J. H. Sun, K. L. Lo, Transient Stability Real-Time Prediction for MultiMachine Power Systems by Using Observation, in Proc. of IEEE Region 10 Conference on Computer, Communication, Control and Power Engineering, vol. 5, pp. 217-221, 1993.

[4] Guoqing Li, Fujun Sun, Qiang Ren, "Real-Time Prediction and Control Method for Transient Stability of Multi-Machine Power System Based on Outside Observation”, Power System Technology, vol. 19, no. 1, pp. 17-22, 1995 (in Chinese).

[5] F. F. Sun, T. S. Bi, H. Y. Li, and etc. "The Perturbed Trajectories Prediction Method Based on Wide-Area Measurement System", in Proc. of IEEE PES Transmission and Distribution Conference and Exhibition, pp. 1467-1471, 2006.

[6] F. F. Song, T. S. Bi, Q. X. Yang. "Perturbed Trajectory Prediction Method based on Simplified Power System Model", in Proc. of IEEE PES Power System Conference and Exposition, pp. 1418-1422, 2006.

[7] Yujing Wang, Jilai Yu, "Real Time Transient Stability Prediction of Multi-Machine System Based on Wide Area Measurement", in Proc. of Asia-Pacific Power and Energy Engineering Conference, pp. 1-4, 2009.

[8] K. Yamashita, H. Kameda. Out-of-Step Prediction Logic for Wide-Area Protection Based on an Autoregressive Model, in Proc. of IEEE PES Power Systems Conference and Exposition, vol. 1, pp. 307-312, 2004.

[9] A. G. Bahbah, A. A. Girgis, "New Method for Generators' Angles and Angular Velocities Prediction for Transient Stability Assessment of Multimachine Power Systems Using Recurrent Artificial Neural Network". IEEE Trans. on Power Systems, vol. 19, no. 2, pp. 10151022, 2004.

[10] I. Kamwa, S. R. Samantaray, G. Joos, "Development of Rule-Based Classifiers for Rapid Stability Assessment of Wide-Area PostDisturbance Records", IEEE Trans. on Power Systems, vol.24, no. 1, pp. 258-270, 2009.

[11] Zhian Zhong, Chunchun Xu, Li Zhang, etc, "Power System Frequency Monitoring Network(FNET) Implementation", IEEE Trans. on Power Systems, vol. 20, no. 4, pp. 1914-1921, 2005.

[12] Lennart Ljung, "System Identification: Theory for the User", Englewood Cliffs: Prentice-Hall, Inc. 1987. 